\title{
Dissipation of shear-free turbulence near boundaries
}

Article

Accepted Version

Teixeira, M. A. C. and Belcher, S. E. (2000) Dissipation of shear-free turbulence near boundaries. Journal Of Fluid Mechanics, 422. pp. 167-191. ISSN 0022-1120 doi: https://doi.org/10.1017/S002211200000149X Available at https://centaur.reading.ac.uk/29256/

It is advisable to refer to the publisher's version if you intend to cite from the work. See Guidance on citing.

To link to this article DOI: http://dx.doi.org/10.1017/S002211200000149X

Publisher: Cambridge University Press

All outputs in CentAUR are protected by Intellectual Property Rights law, including copyright law. Copyright and IPR is retained by the creators or other copyright holders. Terms and conditions for use of this material are defined in the End User Agreement.

\section{www.reading.ac.uk/centaur}

\section{CentAUR}

Central Archive at the University of Reading

Reading's research outputs online 


\title{
Dissipation of shear-free turbulence near boundaries
}

\author{
By M. A. C. TEIX EIR A \\ AND S. E. BELCHER
}

Department of Meteorology, University of Reading, Earley Gate, PO Box 243, Reading RG6 6BB, UK

(Received ?? and in revised form ??)

The rapid-distortion model of Hunt \& Graham (1978) for the initial distortion of turbulence by a flat boundary is extended to account fully for viscous processes. Two types of boundary are considered: a solid wall and a free surface. The model is shown to be formally valid provided two conditions are satisfied. The first condition is that time is short compared with the decorrelation time of the energy-containing eddies, so that nonlinear processes can be neglected. The second condition is that the viscous layer near the boundary, where tangential motions adjust to the boundary condition, is thin compared with the scales of the smallest eddies. The viscous layer can then be treated using thin-boundary-layer methods. Given these conditions, the distorted turbulence near the boundary is related to the undistorted turbulence, and thence profiles of turbulence dissipation rate near the two types of boundary are calculated and shown to agree extremely well with profiles obtained by Perot \& Moin (1993) by direct numerical simulation. The dissipation rates are higher near a solid wall than in the bulk of the flow because the noslip boundary condition leads to large velocity gradients across the viscous layer. In contrast, the weaker constraint of no stress at a free surface leads to the dissipation rate close to a free surface actually being smaller than in the bulk of the flow. This explains why tangential velocity fluctuations parallel to a free surface are so large. In addition we show that it is the adjustment of the large energy-containing eddies across the viscous layer that controls the dissipation rate, which explains why rapid-distortion theory can give quantitatively accurate values for the dissipation rate. We also find that the dissipation rate obtained from the model evaluated at the time when the model is expected to fail actually yields useful estimates of the dissipation obtained from the direct numerical simulation at times when the nonlinear processes are significant. We conclude that the main role of nonlinear processes is to arrest growth by linear processes of the viscous layer after about one large-eddy turnover time.

\section{Introduction}

Turbulence at finite Reynolds number is always subject to dissipation by viscosity. And when a turbulent flow is unforced, it decays in a time that scales on the decorrelation time for the energy-containing turbulent fluctuations (Batchelor, 1953). Hence the dissipation rate, together with the turbulent kinetic energy, provide a time scale for the evolution of the turbulent flow. Parameterisation of the dissipation rate is therefore at the heart of many Reynolds-averaged models of turbulent flows. Nevertheless, there are at present few calculations of the dissipation rate, a notable exception being the study of Hallbäck et al. (1990) where, however, the effect of boundaries is not considered. In the present study we develop an exact calculation of the dissipation rate in the rapid-distortion limit.

The study of the dissipation rate of turbulence presented here was motivated by a general desire to understand better turbulence near a free surface, and has practical importance for transfer of sparingly soluble gases into the liquid that is in turbulent flow (Theofanous, 1984). Now, free surfaces cannot support mean shear, since by definition a 'free' surface must support zero surface stress. Hence, we focus here on turbulence near a free surface in the absence of mean shear.

Shear-free turbulence near boundaries has been the subject of extensive study following the pioneering laboratory measurements of Uzkan \& Reynolds (1967) and Thomas \& Hancock (1977). The definitive theoretical study of Hunt \& Graham (1978), based on rapid-distortion theory, shows 
that the boundary leads to a layer of blocking, where the normal velocity fluctuations are brought to zero, and to a thinner viscous layer, where the tangential velocity fluctuations adjust to the condition at the boundary. More recently, Pan \& Banerjee (1995) carried out direct numerical simulations of free-surface turbulence in channel flow, identifying 3 main flow structures: upwellings, downwellings and persistent vortices 'attached' to the free surface. Perot \& Moin (1995a) and Walker et al. (1996) have reported direct numerical simulations of shear-free boundary layers, which provide inter alia data for profiles of the rate of dissipation of the turbulence and other correlations. These studies reveal significant differences between shear-free boundary layers near solid walls and free surfaces, such as the higher tangential Reynolds stress and lower dissipation near a free surface. An objective of the present study has been to clarify and quantify the mechanisms responsible for these differences.

In this paper the rapid-distortion model developed by Hunt \& Graham (1978) for the initial response of initially homogeneous turbulence to the sudden imposition of a boundary is further developed to account fully for the viscous effects of free surfaces and solid walls. The dissipation rate of the turbulence is then calculated from the model. There is a significant question over whether or not rapid-distortion theory (hereafter RDT) can be usefully used to calculate dissipation. The reason is that the crucial assumption used in RDT of neglecting nonlinear interactions within the turbulence is usually justified, following the original RDT paper of Batchelor \& Proudman (1954), by scaling estimates based on the dynamics of the energy-containing eddies; dissipation, in contrast, is usually associated with the smallest eddies, for which nonlinear processes become important at earlier times. The calculations presented here show how and why RDT can be used to provide useful estimates of dissipation.

The remainder of the paper is organised as follows. In $\S 2$ a scaling analysis of the equations of motion is presented, which identifies some conditions for the validity of the RDT model. In $\S 3$ the response of a single Fourier mode of the turbulence to a solid wall and to a free surface is calculated. These Fourier modes are integrated over a spectrum in $\S 4$ to obtain statistics of the flow near the boundary. In $\S 5$ results are presented for the Reynolds stress and dissipation rate and compared with the DNS data of Perot \& Moin (1993, 1995a, 1995b). Finally, in $\S 6$, conclusions are presented.

\section{Scaling the equations of motion}

Following Hunt \& Graham (1978), we study the following model problem. For times $t<0$, there everywhere exists homogeneous turbulence, characterised by an integral length scale, $L$, and a root mean square velocity scale, $U$. At time $t=0$, a boundary is introduced at $z=0$ and we investigate the initial response and subsequent evolution of the turbulence for $t>0+$ below the interface $z \leq 0$. Two types of boundary are considered: a solid wall and a free surface. A solid wall imposes the condition that $u_{\alpha}=w=0$ at $z=0$, where $u_{\alpha}(\alpha=1$ or 2$)$ are the tangential velocity components and $w$ is the normal velocity component. We consider the distortion of the turbulence by a free surface in the limit of either strong gravity, $g L / U^{2} \gg 1$, or strong surface tension, $T /\left(\rho L U^{2}\right) \gg 1$, whence the interface remains nearly flat. The free surface then imposes the conditions that the surface is stress free, $\tau_{\alpha}=\mu \partial u_{\alpha} / \partial z=0$ (for $\alpha=1$ or 2), and vanishing normal velocity, $w=0$. Far below the boundary, the turbulence tends to the homogeneous form it took before insertion of the boundary.

As shown originally by Hunt \& Graham (1978), flow in the vicinity of the boundary has a two-layer structure. Following Hunt \& Graham, the thickest layer is here called the source layer. This layer is associated with the kinematic blocking effect of the boundary, wherein irrotational motions are induced to bring the normal component of the fluid velocity to zero at the boundary. The source layer responds immediately to the introduction of the boundary and has thickness and velocity scales $L$ and $U$, the scales of the turbulence far below the boundary. The thinner innermost layer, called here the viscous layer, is associated with the viscous effect of the boundary, and is where rotational motions are induced to adjust the tangential motions to the surface boundary condition: in the case of a solid wall the tangential velocity is brought to zero, or in the case of a free surface the tangential stress is brought to zero. The viscous layer is characterised by velocity and length scales determined by its own internal dynamics, so that immediately after the boundary is introduced the viscous layer has zero thickness; it then grows with time as momentum is diffused by molecular viscosity away from the boundary. 
For the purposes of scaling the equations of motion, it is useful to split the turbulent velocity field into three components: the velocity field associated with the homogeneous turbulence in the absence of the boundary, $u_{i}^{(H)}$; the velocity field induced in the source layer, $u_{i}^{(S)}$; and the velocity field induced in the viscous layer, $u_{i}^{(V)}$; namely

$$
u_{i}=u_{i}^{(H)}+u_{i}^{(S)}+u_{i}^{(V)}, \quad i=1,2,3 .
$$

According to the description given above, both $u_{i}^{(H)}$ and $u_{i}^{(S)}$ scale as $U$ and vary over the integral length scale $L$. The depth of the viscous layer scales as $\delta(t)$ and the velocity there varies parallel to the flat interface over the scale $L$. The tangential components of the velocity in the viscous layer, $u_{\alpha}^{(V)}(\alpha=1$ or 2$)$, have magnitudes that are determined from the additional boundary condition at the interface. Hence for a solid wall the no-slip boundary condition yields $u_{\alpha}^{(V)}=O(U)(\alpha=1$ or 2 ), so that by continuity $w^{(V)}=O(U \delta / L)$. And for a free surface the no-stress boundary condition, $\partial u_{\alpha} / \partial z=0$, yields $u_{\alpha}^{(V)}=O(U \delta / L)$, so that by continuity $w^{(V)}=O\left(U \delta^{2} / L^{2}\right)$. The free surface evidently leads to a weaker constraint on the turbulence and hence induces smaller velocities in the viscous layer.

In order to scale the dynamical equations controlling these velocity components, it is helpful to define two time scales. The first is the time scale for turbulent velocity fluctuations to decorrelate, $T_{L}=L / U$. The second is the viscous time scale, which is a measure of the time it takes for the viscous layer to grow to a thickness $\delta$, and is defined as $T_{\nu}=\delta^{2} / \nu$. We consider here the initial development of the turbulence after insertion of the boundary and hence we consider times such that $T_{\nu} \ll T_{L}$. This limit can be expressed in terms of the Reynolds number, $R e=U L / \nu$, and the ratio of the thickness of the viscous layer to the integral length scale of the turbulence, $\delta^{\prime}=\delta / L$ to yield $R e \delta^{\prime 2} \ll 1$. When the boundary is introduced instantaneously at time $t=0$, the viscous layer initially grows as $\delta \sim(\nu t)^{\frac{1}{2}}$ and $T_{\nu} \sim t$; however, it is useful to retain the designation $T_{\nu}$ in order to emphasise that the scalings of the viscous layer depend on the thickness of that layer and not explicitly on time.

On substituting the decomposition of the velocity into components (2.1), and on using their scalings described above, it is found that, in the limit that $T_{\nu} \ll T_{L}$, flow near either a solid boundary or a free surface is described by a simplified form of the vorticity equation, namely,

$$
\frac{\partial \omega_{\alpha}^{(V)}}{\partial t}=\nu \frac{\partial^{2} \omega_{\alpha}^{(V)}}{\partial z^{2}}
$$

or

$$
\frac{\partial}{\partial t}\left(\frac{\partial u_{\alpha}^{(V)}}{\partial z}\right)=\nu \frac{\partial^{2}}{\partial z^{2}}\left(\frac{\partial u_{\alpha}^{(V)}}{\partial z}\right), \quad \alpha=1,2
$$

(cf. Hunt \& Graham, 1978). The tangential vorticity budget is therefore dominated by changes induced by viscous diffusion. Inviscid processes, such as vortex stretching, are negligibly small in this limit. Hence the vorticity changes only in the viscous layer, since this layer is by definition the layer of fluid affected by viscous diffusion. We also conclude, as did Hunt \& Graham (1978), that in the source layer the vorticity is unaffected by insertion of the boundary and hence perturbations to the velocity there, $u_{i}^{(S)}$, are irrotational and can be described by a velocity potential, such that $u_{i}^{(S)}=\partial \phi^{(S)} / \partial x_{i}$. The physical reason for this is that the blocking effect of the boundary is felt instantaneously, whereas vorticity generated by inviscid nonlinear processes takes a time of order $T_{L}$ to become important. Therefore, when $t \ll T_{L}$, the velocity perturbation $u_{i}^{(S)}$ induced by the boundary is essentially irrotational. Since initially $T_{\nu} \sim t$, our scalings show that both the appearance of an irrotational velocity component in the source layer due to blocking by the boundary and tangential vorticity generation by viscous processes in the viscous layer are more important than vorticity generation by inviscid processes in the limit $T_{\nu} \ll T_{L}$. Hence, the criteria for both these approximations turn out to be the same in practice, although the scaling of the viscous layer is based on $T_{\nu}$, whereas the scaling of the source layer is based explicitly on time.

The tangential momentum equation may be scaled in a similar way and, if $T_{\nu} \ll T_{L}$, it is found to take the simplified form

$$
\frac{\partial u_{\alpha}^{(V)}}{\partial t}=\nu \frac{\partial^{2} u_{\alpha}^{(V)}}{\partial z^{2}}, \quad \alpha=1,2
$$


for a solid wall. So, to this approximation, not only does the inviscid part of the flow, $u_{i}^{(H)}+u_{i}^{(S)}$, have constant vorticity but it also has constant velocity. Thus, $u_{i}^{(H)}$ and $u_{i}^{(S)}$ are approximately steady compared with the viscous part of the flow, $u_{i}^{(V)}$.

The tangential velocity components, $u_{\alpha}^{(V)}$, exist inside the viscous layer so that the boundary condition on tangential velocity is satisfied. These tangential motions vary along the boundary and generate regions of convergence and divergence and hence a normal velocity component, of order $u_{\alpha}^{(V)} \delta / L$, at the outer edge of the viscous layer. These motions slightly modify the flow in the source layer. The corresponding corrections are treated by expanding the solutions for $u_{i}^{(S)}$ and $u_{i}^{(V)}$ in power series of the small parameter $\delta^{\prime}=\delta / L$, for example

$$
u^{(S)}=u^{(S 0)}+u^{(S 1)}+u^{(S 2)}+\ldots, \quad u^{(V)}=u^{(V 0)}+u^{(V 1)}+u^{(V 2)}+\ldots,
$$

where the powers of $\delta^{\prime}$ have been incorporated into the terms of the series. In the following, the series for $u_{\alpha}^{(V)}(\alpha=1$ or 2$)$ and $u_{i}^{(S)}$ will be calculated to second order in $\delta^{\prime}$ in the case of a solid wall and to third order in $\delta^{\prime}$ in the case of a free surface. Higher order terms are affected by nonlinear processes and have been neglected. A detailed justification of this procedure is presented in $\S 4.2$. The series expansion of the normal velocity, $w^{(V)}$, is truncated at one order higher than those of the tangential components. This is done so that the series conserve mass exactly, which is important to do if the Reynolds stresses and the dissipation rates are to have the correct behaviour near the boundary. The price paid is that the solutions have small errors far from the boundary, but these are deemed less serious, since it is the near-wall region that is of interest here.

\section{Distortion of a single Fourier component of velocity fluctuation}

The model developed in the previous section, based on the diffusion equations (2.4) and (2.3), is now solved for the cases of a solid wall and a free surface, respectively. Here the modal solutions are calculated for the Fourier amplitudes of the flow variables. These modal solutions will be integrated over the spectrum of the turbulence in $\S 4$.

\subsection{Solid wall}

We begin by considering the effects of a solid wall. If the series (2.5) are substituted into the tangential momentum equation, then, to the 3 lowest orders in $\delta^{\prime}$, the equation for velocity in the viscous layer becomes

$$
\frac{\partial u_{\alpha}^{(V j)}}{\partial t}=\nu \frac{\partial^{2} u_{\alpha}^{(V j)}}{\partial z^{2}}
$$

where $\alpha=1$ or 2 and $j=0,1$ or 2 . Mass conservation then determines the normal component of velocity.

In the source layer, where as argued above $u_{i}^{(S)}=\partial \phi^{(S)} / \partial x_{i}$, mass conservation requires that the velocity potential $\phi^{(S)}$ satisfies the Laplace equation, which means that for $j=0,1$ or 2

$$
\nabla^{2} \phi^{(S j)}=0 \text {. }
$$

The boundary conditions are as follows. The flow tends to the original homogeneous turbulence, $u_{i}^{(H)}$, far below the boundary. Hence both $\phi^{(S j)}$ and $u_{i}^{(V j)}$ tend to zero far below the boundary. The normal and tangential velocity components vanish at the boundary. Finally, the initial condition states that the initial vorticity equals the vorticity in the homogeneous turbulence. Hence $u_{i}^{(V)}$ vanishes everywhere at $t=0+$.

These equations are solved, following Hunt \& Graham (1978), by noting firstly that the homogeneous turbulent velocity $u_{i}^{(H)}$ may be expressed as a superposition of Fourier modes,

$$
u_{i}^{(H)}(\boldsymbol{x})=\iiint \hat{u}_{i}^{(H)}(\boldsymbol{k}) \mathrm{e}^{\mathrm{i} k \cdot x} \mathrm{~d} k_{1} \mathrm{~d} k_{2} \mathrm{~d} k_{3}, \quad i=1,2,3,
$$

where $\hat{u}_{i}^{(H)}(\boldsymbol{k})$ is the Fourier amplitude and $\boldsymbol{k}=\left(k_{1}, k_{2}, k_{3}\right)$ is the wavenumber vector. Note secondly that, due to the geometry of the problem, $u_{i}^{(V)}$ and $u_{i}^{(S)}$ (and hence also $\phi^{(S)}$ ) are homogeneous in the directions tangential to the boundary, and so they can equally be expressed 
as a superposition of Fourier modes along those directions:

$$
\begin{aligned}
\phi^{(S)}(\boldsymbol{x}, t) & =\iint \hat{\phi}^{(S)}\left(k_{1}, k_{2}, z, t\right) \mathrm{e}^{\mathrm{i}\left(k_{1} x+k_{2} y\right)} \mathrm{d} k_{1} \mathrm{~d} k_{2} \\
u_{i}^{(V)}(\boldsymbol{x}, t) & =\iint \hat{u}_{i}^{(V)}\left(k_{1}, k_{2}, z, t\right) \mathrm{e}^{\mathrm{i}\left(k_{1} x+k_{2} y\right)} \mathrm{d} k_{1} \mathrm{~d} k_{2} .
\end{aligned}
$$

Hence, since the equations are linear, the response of one Fourier mode only needs to be considered.

The solution for the Fourier amplitude of the velocity potential in the source layer obtained by solving (3.2) is found to be

$$
\hat{\phi}^{(S)}=-\int \frac{\hat{w}^{(H)}}{k_{12}}\left[1-\frac{\delta}{\pi^{1 / 2}}\left(\mathrm{i} k_{3}-k_{12}\right)\left(1+\frac{\delta k_{12}}{\pi^{1 / 2}}\right)\right] \mathrm{e}^{k_{12} z} \mathrm{~d} k_{3},
$$

where $k_{12}=\left(k_{1}^{2}+k_{2}^{2}\right)^{\frac{1}{2}}$, and which is correct up to second order in $\delta^{\prime}$. The zeroth-order part of (3.5) is the well-known inviscid solution of Hunt \& Graham (1978); the corrections of $O\left(\delta^{\prime}\right)$ and $O\left(\delta^{\prime 2}\right)$ are new. The components of $u_{i}^{(S)}$ are easily calculated from the potential $\phi^{(S)}$.

The viscous solution may be found by solving (3.1) subject to the boundary and initial conditions. The Fourier amplitude of the tangential velocity is

$$
\hat{u}_{\alpha}^{(V)}=-\int\left\{\hat{u}_{\alpha}^{(V)}-\frac{\mathrm{i} k_{\alpha}}{k_{12}} \hat{w}^{(H)}\left[1-\frac{\delta}{\pi^{1 / 2}}\left(\mathrm{i} k_{3}-k_{12}\right)\left(1+\frac{\delta k_{12}}{\pi^{1 / 2}}\right)\right]\right\}\left[\operatorname{erf}\left(\frac{z}{\delta}\right)+1\right] \mathrm{d} k_{3},
$$

where $\alpha=1$ or 2 and $\delta=2(\nu t)^{\frac{1}{2}}$. The corresponding Fourier amplitude of the normal velocity is

$$
\begin{aligned}
\hat{w}^{(V)}= & -\int \delta \hat{w}^{(H)}\left(\mathrm{i} k_{3}-k_{12}\right)\left\{\left(1+\frac{\delta k_{12}}{\pi^{1 / 2}}+\frac{\delta^{2} k_{12}^{2}}{\pi}\right)\left[\frac{z}{\delta}\left(\operatorname{erf}\left(\frac{z}{\delta}\right)+1\right)+\frac{1}{\pi^{1 / 2}} \mathrm{e}^{-z^{2} / \delta^{2}}\right]\right. \\
& \left.-\frac{\delta^{2} k_{12}^{2}}{\pi^{3 / 2}}\right\} \mathrm{d} k_{3} .
\end{aligned}
$$

\subsection{Free surface}

The solutions are now calculated for turbulence near a free surface. If in the viscous layer the series (2.5) are substituted into (2.3) then for $j=0,1,2$ or 3 the tangential velocity components satisfy

$$
\frac{\partial}{\partial t} \frac{\partial u_{\alpha}^{(V j)}}{\partial z}=\nu \frac{\partial^{2}}{\partial z^{2}} \frac{\partial u_{\alpha}^{(V j)}}{\partial z}
$$

where $\alpha=1$ or 2 , and, as for the solid wall, the vertical velocity component is obtained from continuity. In the source layer the velocity potential satisfies the Laplace equation (3.2) as for a solid wall.

The boundary conditions ensure that the turbulent velocity tends to the homogeneous turbulence far below the boundary, and that at the boundary, $z=0$, the normal velocity component and the normal derivative of the tangential velocity components (and hence also the tangential stress) vanish.

The solution for the Fourier amplitude of the velocity potential in the source layer, correct to third order in $\delta^{\prime}$, is found to be

$$
\hat{\phi}^{(S)}=-\int \frac{\hat{w}^{(H)}}{k_{12}}\left(1+\frac{\delta^{2} k^{2}}{4}\right) \mathrm{e}^{k_{12} z} \mathrm{~d} k_{3} .
$$

Again, the zeroth-order part of (3.9) is the well-known inviscid solution of Hunt \& Graham (1978) and is identical to the solution calculated for a solid wall. The corrections, which arise due to vertical motions induced in the viscous layer, are new and are different from the corrections to $\hat{\phi}^{(S)}$ obtained for a solid wall because the solutions in the viscous layer are different. The velocity in the source layer is easily calculated from the velocity potential $\phi^{(S)}$.

The viscous solution for the amplitude of the tangential velocity is

$$
\hat{u}_{\alpha}^{(V)}=-\int \delta\left[\mathrm{i} k_{3} \hat{u}_{\alpha}^{(H)}-\mathrm{i} k_{\alpha} \hat{w}^{(H)}\left(1+\frac{\delta^{2} k^{2}}{4}\right)\right]\left[\frac{z}{\delta}\left(\operatorname{erf}\left(\frac{z}{\delta}\right)+1\right)+\frac{1}{\pi^{1 / 2}} \mathrm{e}^{-z^{2} / \delta^{2}}\right] \mathrm{d} k_{3},
$$


where $\alpha=1$ or 2 . The corresponding amplitude of the normal velocity is

$$
\begin{aligned}
\hat{w}^{(V)}= & \int \delta^{2} k^{2} \hat{w}^{(H)}\left\{( 1 + \frac { \delta ^ { 2 } k _ { 1 2 } ^ { 2 } } { 4 } ) \left[\frac{z^{2}}{2 \delta^{2}}\left(\operatorname{erf}\left(\frac{z}{\delta}\right)+1\right)+\frac{z}{2 \pi^{1 / 2} \delta} \mathrm{e}^{-z^{2} / \delta^{2}}\right.\right. \\
& \left.\left.+\frac{1}{4}\left(\operatorname{erf}\left(\frac{z}{\delta}\right)+1\right)\right]-\frac{\delta^{2} k_{12}^{2}}{16}\right\} \mathrm{d} k_{3} .
\end{aligned}
$$

The differences between the solutions for a solid wall and a free surface will be illustrated later when statistics of the flow have been calculated.

\section{Integration of Fourier amplitudes to obtain statistics}

In $\S 3$, solutions were found for how the Fourier amplitudes of the flow variables respond to the presence of the boundary. These modal solutions are now integrated over a spectrum of wavenumbers in order to understand the response of turbulence consisting of fluctuations on a wide range of scales. In this way the turbulence near the boundary is related to the spectrum of the undistorted turbulence far from the boundary. Firstly, to validate the RDT method used here, profiles of the Reynolds stresses are calculated and compared with the profiles that Perot \& Moin $(1993,1995 a)$ obtained by DNS. Then rates of turbulence dissipation are calculated using the model, which have not been calculated hitherto with RDT. Finally, these results are compared with the dissipation rates computed by Perot \& Moin $(1993,1995 b)$.

Statistics of the distorted turbulence are related to statistics of the undistorted turbulence far from the boundary using the framework developed by Hunt (1973). The solution for a single mode of the velocity fluctuation is given by the distorted Fourier amplitude $\hat{u}_{i}\left(k_{1}, k_{2}, z, t\right)$, which is related to the complete velocity field $u_{i}(\boldsymbol{x}, t)$ by

$$
u_{i}(\boldsymbol{x}, t)=\iint \hat{u}_{i}\left(k_{1}, k_{2}, z, t\right) \mathrm{e}^{\mathrm{i}\left(k_{1} x+k_{2} y\right)} \mathrm{d} k_{1} \mathrm{~d} k_{2} .
$$

Since the RDT model is linear, the distorted Fourier amplitude is linearly related to the Fourier amplitude of the undistorted turbulence, $\hat{u}_{i}^{(H)}$, and so can be written

$$
\hat{u}_{i}\left(k_{1}, k_{2}, z, t\right)=\int M_{i k}(\boldsymbol{k}, z, t) \hat{u}_{k}^{(H)}(\boldsymbol{k}) \mathrm{d} k_{3} .
$$

The matrix $M_{i k}$ is extracted from the solutions calculated in $\S 3$ and is listed in the appendix. Statistics of the flow are then calculated on recalling that the Fourier amplitude of the undistorted turbulence is related to the three-dimensional wavenumber spectrum, $\Phi_{i j}^{(H)}$, by

$$
\overline{\hat{u}_{i}^{(H)}(\boldsymbol{k}) \hat{u}_{j}^{(H)}\left(\boldsymbol{k}^{\prime}\right)}=\Phi_{i j}^{(H)}(\boldsymbol{k}) \delta\left(\boldsymbol{k}-\boldsymbol{k}^{\prime}\right),
$$

where the overbar denotes ensemble averaging.

Using (4.1), (4.2) and (4.3), the Reynolds stresses can then be written

$$
\overline{u_{i}^{2}}(z, t)=\iiint M_{i k}^{*} M_{i l} \Phi_{k l}^{(H)} \mathrm{d} k_{1} \mathrm{~d} k_{2} \mathrm{~d} k_{3},
$$

where $i=1,2$ or 3 . The diagonal elements of the Reynolds stress tensor (the velocity variances) are the only non-zero components for the initially isotropic, shear-free turbulence considered here. Accordingly, only the diagonal components of the dissipation tensor are non-zero. These are defined by

$$
\varepsilon_{i i}(z, t)=2 \nu\left[\overline{\left(\frac{\partial u_{i}}{\partial x}\right)^{2}}+\overline{\left(\frac{\partial u_{i}}{\partial y}\right)^{2}}+\overline{\left(\frac{\partial u_{i}}{\partial z}\right)^{2}}\right],
$$

where $i=1,2$ or 3 . The variances of velocity derivatives along the boundary are calculated from the undistorted turbulence by

$$
\overline{\left(\frac{\partial u_{i}}{\partial x_{\alpha}}\right)^{2}}=\iiint k_{\alpha}^{2} M_{i k}^{*} M_{i l} \Phi_{k l}^{(H)} \mathrm{d} k_{1} \mathrm{~d} k_{2} \mathrm{~d} k_{3}
$$

where $i=1,2$ or 3 and $\alpha=1$ or 2 . The variances of derivatives normal to the boundary are 
calculated from the undistorted turbulence by

$$
\overline{\left(\frac{\partial u_{i}}{\partial z}\right)^{2}}=\iiint \frac{\partial M_{i k}^{*}}{\partial z} \frac{\partial M_{i l}}{\partial z} \Phi_{k l}^{(H)} \mathrm{d} k_{1} \mathrm{~d} k_{2} \mathrm{~d} k_{3} .
$$

Thus, the Reynolds stresses and the components of the dissipation rate tensor are calculated from the spectrum of the undistorted turbulence.

In order to proceed further, the undistorted turbulence far from the boundary is supposed to be homogeneous and isotropic. The three-dimensional velocity spectrum is then related to the energy spectrum $E(k)$ by

$$
\Phi_{i j}^{(H)}=\left(\delta_{i j}-\frac{k_{i} k_{j}}{k^{2}}\right) \frac{E(k)}{4 \pi k^{2}},
$$

where the energy spectrum is defined by

$$
\frac{1}{2}\left(\overline{u^{(H) 2}}+\overline{v^{(H) 2}}+\overline{w^{(H) 2}}\right)=\int_{0}^{\infty} E(k) \mathrm{d} k .
$$

Once the form of the energy spectrum is specified, (4.4) and (4.5) can be evaluated to give profiles of the Reynolds stresses and the dissipation rates. The model for the energy spectrum is considered next.

\subsection{The model for the energy spectrum}

In their inviscid RDT calculations, Hunt \& Graham (1978) used the Von Kármán form of the energy spectrum

$$
E(k)=U^{2} L \frac{g_{2}(k L)^{4}}{\left(g_{1}+(k L)^{2}\right)^{17 / 6}},
$$

where $g_{1}$ and $g_{2}$ are dimensionless constants. This form has an inertial subrange (where the spectrum decays as $k^{-5 / 3}$ ) that extends to $k \rightarrow \infty$, corresponding to infinite Reynolds number. This spectrum is appropriate for calculating quantities that receive their greatest contribution from the energy containing part of the spectrum, such as the Reynolds stresses, but it is not appropriate for calculating quantities that receive a significant contribution from the high wavenumbers, such as the dissipation rate. In fact, because the Von Kármán spectrum does not have a viscous cut off at high wavenumbers, the integrals that give the dissipation rate, (4.6) and (4.7), diverge. Hence, here it is necessary to use a form for the energy spectrum that accounts for the viscous processes at high wavenumbers.

According to Tennekes \& Lumley (1972, p.269), for large Reynolds number, the energy spectrum at high wavenumbers is reasonably approximated by

$$
E(k) \sim \alpha \varepsilon^{2 / 3} k^{-5 / 3} \exp \left[-\frac{3}{2} \alpha(k \eta)^{4 / 3}\right],
$$

where $\alpha$ is a constant approximately equal to 1.5 and the Kolmogorov microscale, $\eta$, is given by

$$
\eta=\left(\frac{\nu^{3}}{\varepsilon}\right)^{1 / 4}
$$

where $\varepsilon=1 / 2\left(\varepsilon_{11}+\varepsilon_{22}+\varepsilon_{33}\right)$ is the isotropic dissipation rate in homogeneous turbulence.

Hence, an obvious extension of (4.10) for finite Reynolds numbers is

$$
E(k)=U^{2} L \frac{g_{2}(k L)^{4}}{\left(g_{1}+(k L)^{2}\right)^{17 / 6}} \exp \left[-\frac{3}{2} \alpha(k \eta)^{4 / 3}\right] .
$$

This form must be made to satisfy 3 constraints in order to become completely defined. The first constraint results from the definition of root mean square velocity, $U^{2}=\overline{u_{i}^{(H) 2}}$, with $i=1,2$ or 3. Since the total kinetic energy of the homogeneous turbulence is given by the integral over all wavenumbers of $E(k)$ (see $(4.9)$ ), then

$$
\int_{0}^{\infty} E(k) \mathrm{d} k=\frac{3}{2} U^{2}
$$

The second constraint is that the length scale $L$ in (4.13) is defined as the longitudinal integral 
length scale, so that

$$
L=\frac{\int_{0}^{\infty} \overline{u^{(H)}(x, y, z) u^{(H)}(x+r, y, z)} \mathrm{d} r}{\overline{u^{(H) 2}}}=\frac{3 \pi}{4} \frac{\int_{0}^{\infty} k^{-1} E(k) \mathrm{d} k}{\int_{0}^{\infty} E(k) \mathrm{d} k} .
$$

The third constraint is given by the definition of viscous dissipation in homogeneous isotropic turbulence, namely

$$
\varepsilon=2 \nu \int_{0}^{\infty} k^{2} E(k) \mathrm{d} k .
$$

If these constraints are cast into dimensionless form they can be used to determine the unknown coefficients in (4.13).

If the dimensionless wavenumber is defined as $k^{\prime}=k L$ then equation (4.12) shows that $\eta / L$ is

$$
\frac{\eta}{L}=R e^{-3 / 4}\left(\frac{\varepsilon L}{U^{3}}\right)^{-1 / 4}=R e^{-3 / 4} \varepsilon^{-1 / 4} .
$$

where $\varepsilon^{\prime}$ is the dimensionless dissipation rate. The dimensionless energy spectrum, defined as $E^{\prime}\left(k^{\prime}\right)=E(k) /\left(U^{2} L\right)$, is then

$$
E^{\prime}\left(k^{\prime}\right)=\frac{g_{2} k^{\prime 4}}{\left(g_{1}+k^{\prime 2}\right)^{17 / 6}} \exp \left[-\frac{3}{2} \alpha R e^{-1} \varepsilon^{\prime-1 / 3} k^{\prime 4 / 3}\right] .
$$

The three constraints (4.14), (4.15) and (4.16) then take the dimensionless form

$$
\int_{0}^{\infty} E^{\prime}\left(k^{\prime}\right) \mathrm{d} k^{\prime}=\frac{3}{2}, \quad \int_{0}^{\infty} k^{\prime-1} E^{\prime}\left(k^{\prime}\right) \mathrm{d} k^{\prime}=\frac{2}{\pi}, \quad \int_{0}^{\infty} k^{\prime 2} E^{\prime}\left(k^{\prime}\right) \mathrm{d} k^{\prime}=\frac{1}{2} R e \varepsilon^{\prime} .
$$

Hence, for a given value of $\alpha$ and a given Reynolds number, the dimensionless spectrum (4.18) contains 3 unknown parameters: $g_{1}, g_{2}$ and $\varepsilon^{\prime}$, which can be determined uniquely by solving the implicit equation set (4.19).

The numerical evaluation of the integrals that determine the dissipation rate is made simpler if this form of the spectrum (4.18) is approximated by truncating at a finite wavenumber, $k_{\max }$, namely

$$
\begin{aligned}
& E^{\prime}\left(k^{\prime}\right)=\frac{g_{2} k^{\prime 4}}{\left(g_{1}+k^{2}\right)^{17 / 6}} \quad \text { if } \quad k^{\prime} \leq k_{\max }^{\prime} \\
& E^{\prime}\left(k^{\prime}\right)=0 \quad \text { if } \quad k^{\prime}>k_{\text {max }}^{\prime} .
\end{aligned}
$$

where $k_{\text {max }}^{\prime}=k_{\max } L$ is the dimensionless cutoff wavenumber. Here $k_{\max }^{\prime}$ is determined by requiring that the dimensionless dissipation rate is correct. In the limit of high Re the high wavenumbers dominate the dissipation rate, hence the full spectrum (4.18) gives

$$
\int_{0}^{\infty} k^{\prime 2} E^{\prime}\left(k^{\prime}\right) \mathrm{d} k^{\prime} \approx \int_{0}^{\infty} g_{2} k^{\prime 1 / 3} \exp \left[-\frac{3}{2} \alpha R e^{-1} \varepsilon^{\prime-1 / 3} k^{4 / 3}\right] \mathrm{d} k^{\prime}=\frac{g_{2}}{2 \alpha} R e \varepsilon^{\prime 1 / 3} .
$$

whereas the truncated spectrum gives

$$
\int_{0}^{k_{\text {max }}^{\prime}} k^{\prime 2} E^{\prime}\left(k^{\prime}\right) \mathrm{d} k^{\prime} \approx \int_{0}^{k_{\text {max }}^{\prime}} g_{2} k^{\prime 1 / 3} \mathrm{~d} k^{\prime}=\frac{3}{4} g_{2} k_{\text {max }}^{\prime 4 / 3} .
$$

So, in this regime, the cutoff wavenumber is obtained by comparing (4.22) and (4.21):

$$
k_{\text {max }}^{\prime}=\left(\frac{2}{3 \alpha}\right)^{3 / 4} \varepsilon^{1 / 4} R e^{3 / 4} .
$$

This procedure yields $k_{\max } \eta=[2 /(3 \alpha)]^{3 / 4} \approx 0.54$, as found by Tennekes and Lumley. Hence $k_{\max }$ is of order $1 / \eta$.

Figure 1 shows plots of the dimensionless profiles of the truncated spectrum (4.20) and full spectrum (4.18) for $R e=70$ and $\alpha=1.5$. Figure 2 displays the dependence of dimensionless dissipation on the Reynolds number for the complete spectrum and the truncated spectrum, together with values computed with DNS by Jiménez et al. (1993) and Wang et al. (1996). In the complete spectrum, $\alpha=2$ (chosen so as to optimise the fit), whereas in the truncated spectrum, $\alpha=1.5$, as suggested by Tennekes and Lumley (1972). While it is clear that the complete spectrum gives an 
excellent fit to the data, the truncated spectrum also reproduces the general trend quite well for $R e>70$. In what follows, all calculations will be performed using the truncated spectrum (with $\alpha=1.5$ ), because this considerably shortens the time required for numerical integration.

\subsection{Validity of the model}

There are two conditions that must be satisfied if the current model is to be valid. Firstly, nonlinear processes in the turbulence have to be negligible compared with the diffusive growth of the viscous layer. As shown in $\S 2$, this condition is met provided $R e \delta^{2} \ll 1$. Secondly, the small effect of viscosity was included by treating it as a small perturbation to the inviscid processes. Hence the velocity components were expanded as series in (2.5), and these series were truncated after the first three terms. Now, when statistics are calculated from the modal solutions for velocity, the magnitude of the neglected terms depends on the distribution of energy within the spectrum. Hence, the condition required for these truncated expansions to be valid will now be determined.

The solution for $M_{i k}$ given in the appendix, which is in the form of a power series in $\delta$, together with the expression for the Reynolds stresses (4.4) show that, for example, the streamwise Reynolds stress is also a series in $\delta$, namely

$$
\overline{u^{2}}=\int_{0}^{\infty} g_{0}(k, z) E(k) \mathrm{d} k+\delta \int_{0}^{\infty} g_{1}(k, z) E(k) \mathrm{d} k+\delta^{2} \int_{0}^{\infty} g_{2}(k, z) E(k) \mathrm{d} k+\ldots,
$$

where the functions $g_{n}(k, z)=O\left(k^{n}\right)$. The peak of the spectrum dominates in the first integral of (4.24), which is therefore independent of $k_{\max }$, but the high-wavenumber tail of the spectrum dominates the second and third integrals. Having in mind that $E(k) \propto k^{-5 / 3}$ at high wavenumbers, these integrals can be estimated to scale as

$$
\begin{aligned}
& \int_{0}^{k_{\text {max }}} g_{0}(k, z) E(k) \mathrm{d} k=O\left(U^{2}\right) \\
& \int_{0}^{k_{\text {max }}} g_{1}(k, z) E(k) \mathrm{d} k=O\left(U^{2} L^{-2 / 3} \int_{0}^{k_{\text {max }}} k k^{-5 / 3} \mathrm{~d} k\right)=O\left(U^{2} L^{-2 / 3} k_{\text {max }}^{1 / 3}\right) \\
& \int_{0}^{k_{\text {max }}} g_{2}(k, z) E(k) \mathrm{d} k=O\left(U^{2} L^{-2 / 3} \int_{0}^{k_{\text {max }}} k^{2} k^{-5 / 3} \mathrm{~d} k\right)=O\left(U^{2} L^{-2 / 3} k_{\text {max }}^{4 / 3}\right) .
\end{aligned}
$$

And the series expansion for the Reynolds stress becomes

$$
\overline{u^{2}} / U^{2}=A_{0}+A_{1} k_{\text {max }}^{\prime 1 / 3} \delta^{\prime}+A_{2} k_{\text {max }}^{\prime 4 / 3} \delta^{2}+\ldots,
$$

where the coefficients $A_{n}=O(1)$. This expansion is asymptotic provided

$$
k_{\max }^{\prime} \delta^{\prime} \ll 1 .
$$

This condition arises physically from the treatment of the viscous layer as a thin boundary layer, i.e. the assumption that variations across the viscous layer, normal to the surface, are much more rapid than variations along the boundary layer, parallel to the surface. The smallest-scale variations in the source layer just outside the viscous layer are determined by the smallest scale in the turbulence, namely $\eta \sim k_{m a x}^{-1}$. Hence this boundary layer approximation is valid only when these smallest scales are much larger than the thickness of the viscous layer.

Since the viscous layer grows initially with time as $\delta=2(\nu t)^{\frac{1}{2}}$, this condition (4.27) together with the definitions of $\delta^{\prime}=\delta / L$ and $k_{\text {max }}^{\prime}$ given in (4.23), give a condition on time, namely

$$
\frac{U t}{L} \ll C R e^{-1 / 2}
$$

where $C=1 / 4(3 \alpha / 2)^{3 / 2} \varepsilon^{-1 / 2} \approx 1$ for $\alpha=1.5$. Hence for very large Reynolds numbers, when the turbulence has very small scales, the model developed here for the dissipation is valid for only short times. We have indeed detected such a dependence on the Reynolds number in the comparisons between the model and the DNS.

If the scaling analysis is carried out again for the velocity fluctuations taking into account the dependence of their magnitude on the spectrum, as implied by (4.26), then it is found that the terms retained in the expansions for the velocity fluctuations are larger than the nonlinear terms that were neglected provided $T_{\nu} \ll T_{L}$, i.e. that $t \ll L / U$. This is the conventional requirement 
for the validity of RDT: in the present flow, the particular form of the spectrum apparently does not affect the form of this approximation.

\subsection{Truncation errors}

Finally, before showing results from the model, we note a practical aspect of implementing the current solutions in calculating statistics of the turbulence near the boundary.

The Reynolds stresses are the square of velocity. So, if the power-series solutions for the velocity are multiplied together, then the highest order terms in this product series, which correspond to the products of the highest order terms of the velocity series, are of higher order than the truncation error. Hence these higher-order terms might be considered negligible. However, the original expansions for the velocity satisfy the boundary conditions at $z=0$ exactly. Hence, if the high-order products are neglected, then the resulting statistics become unreliable very close to the boundary. The truncated and the non-truncated expressions both have the same formal accuracy, since they differ from each other only by terms of the order the truncation error. Therefore, the full products are prefered here as they preserve the exact boundary conditions at $z=0$, and they are adopted everywhere hereafter.

\section{Results and discussion}

In this section, theoretical results obtained with the model developed in the preceding sections will be compared with the DNS data presented in Perot \& Moin (1993) (hereafter referred to as PM93) and Perot \& Moin (1995a, 1995b) (hereafter referred to as PM95a and PM95b, respectively). Two important preliminary considerations must be made before carrying out such a task.

Firstly, the turbulence length and velocity scales defined by these authors are different from those used in this study until now, and have to be related before any comparison is possible. Perot \& Moin normalise their data by defining a velocity scale, $U^{*}$, and length scale, $L^{*}$, based on the values of the kinetic energy $K$ and dissipation rate $\varepsilon$ far from the boundary, i.e.

$$
U^{*}=K^{1 / 2}, \quad L^{*}=\frac{K^{3 / 2}}{\varepsilon} .
$$

They then define a Reynolds number in terms of $L^{*}$ and $U^{*}$ :

$$
R e^{*}=\frac{U^{*} L^{*}}{\nu}=\frac{K^{2}}{\varepsilon \nu}
$$

Noting that the kinetic energy is defined in the present study as $K=(3 / 2) U^{2}$, the two sets of length and velocity scales and Reynolds numbers are found to be related by

$$
U^{*}=\left(\frac{3}{2}\right)^{1 / 2} U, \quad L^{*}=\left(\frac{3}{2}\right)^{3 / 2} \varepsilon^{\prime-1} L, \quad R e^{*}=\frac{9}{4} \varepsilon^{\prime-1} R e .
$$

Secondly, the flow computed by Perot \& Moin is unforced and so the turbulence decays with time. This decay changes some aspects of the flow, although the main features are the same as if the turbulence were stationary, particularly for short times. In the current model, we assume stationary and homogeneous turbulence away from the boundary. Comparison with data obtained where the turbulence decays might be thought to introduce a new limitation on the time interval over which the model is valid. This is not so, however. Viscous decay of energy in the bulk of the flow is just a manifestation of viscous diffusion of momentum or vorticity in the turbulence far from the boundary, a physical process which was shown to be negligible at early times in the scalings of $\S 2$.

PM93 used Reynolds numbers of $R e^{*}=54,134$ and 374 in their numerical simulations of a solid wall and $6.2,54$ and 134 in their simulations of a free surface. The value of $R e^{*}=6.2$ is manifestly too low for the concept of a viscous cutoff to work properly (see $\$ 4.1$ ), and hence this case is not considered here. In terms of the velocity and length scales defined by (5.1), the condition (4.28) for the validity of the analysis of the viscous layer takes the form

$$
\frac{U^{*} t}{L^{*}} \ll \frac{1}{4}\left(\frac{3 \alpha}{2}\right)^{3 / 2} R e^{*-1 / 2}
$$

For Reynolds numbers of 54, 134 and 374 this condition requires that $U^{*} t / L^{*}$ is smaller than 0.11 , 
0.07 and 0.04 respectively. Much of the DNS data presented in PM93, PM95a and PM95b are for times when dissipation and nonlinear processes have become important in the dynamics of the turbulence, and the earliest time that Perot \& Moin show results of is $U^{*} t / L^{*}=0.1$. Therefore comparisons with the theory are made at $U^{*} t / L^{*}=0.1$ for the cases when $R e^{*}=54$ and $R e^{*}=134$. Some qualitative comparisons will be made for later times in $\S 5.3$.

\subsection{Profiles of the Reynolds stresses}

Figure 3 presents profiles of the Reynolds stress for a solid wall and figure 4 profiles for a free surface, at a dimensionless time of $U^{*} t / L^{*}=0.1$ and at Reynolds numbers of 54 and 134. The DNS data presented in figure 3 were taken from figures $15(a, b), 17(a, b)$ of PM95a and those presented in figure 4 were taken from figures $9(a, b), 12(a, b)$ of PM95a. The results from the DNS are compared with the current model accounting for the viscous layer, called here the viscous theory, and with inviscid model results, which are obtained from the present model when the viscous layer is ignored (i.e. $\delta^{\prime}=0$ ), called here the inviscid theory.

Figures $3(a, b)$ show profiles of the tangential Reynolds stress, $\overline{u_{\alpha}^{2}}$, near a solid wall for $R e=54$, 134. The general shape of the DNS curves, as well as the location of their maxima, are well reproduced by the viscous theory. For $R e^{*}=134$ the profile from the viscous theory is in excellent agreement with data, while for $R e^{*}=54$ the theory gives slightly lower values, although the general agreement remains good. In the inviscid theory the stresses increase monotonically towards the boundary, however the viscous processes arrest this increase and lead to a reduction of the stresses, varying approximately as an error function, so that they are zero actually at the surface. Hence there is a maximum in the tangential Reynolds stress at the thickness of the viscous layer, namely $|z| \approx 3(\nu t)^{\frac{1}{2}}$. The maximum value obtained from the model is slightly larger for the larger Reynolds number because, for a given time, the larger the Reynolds number, the thinner the viscous layer. The DNS profiles shown in figures $15(a-c)$ of PM95a show a maximum that increases between $R e^{*}=134$ to $R e^{*}=374$, in agreement with this argument. However, in going from $R e^{*}=54$ to $R e^{*}=134$ the maximum in the DNS profiles actually decreases. This behaviour might be attributed to an imperfect ensemble average for the case of $R e^{*}=54$.

Figures $3(c, d)$ show profiles of the normal Reynolds stress, $\overline{w^{2}}$, near a solid wall. In this case, the magnitude and general behaviour of theory and data agree very well, particularly in figure $3(d)$, but it is also true that viscous and inviscid theory differ much less. The small differences between the theory and the DNS data in figure 3(c) are attributed to an imperfect ensemble averaging, since they are due to a lack of smoothness in the DNS curve. The curve from the viscous theory captures quite well the viscous behaviour in the region immediately adjacent to the boundary, where $\overline{w^{2}} \propto z^{4}$ due to the no-slip boundary condition.

Figures $4(a, b)$ present profiles of the tangential Reynolds stress near a free surface. For the same reason as in figures $3(a, b)$, the quantitative agreement between theory and data is considerably better for a Reynolds number of 134 than for a Reynolds number of 54 (although not as good as in figure $3(b)$ ). As the Reynolds number increases, the viscous model shows a slight increase in the maximum value of the stress, which lies at the free surface. The model shows that this happens because the viscous layer becomes thinner. Surprisingly, the DNS data shows the opposite trend. The enforcement of the boundary condition $\partial \overline{u_{\alpha}^{2}} / \partial z=0$ is evident in the viscous theory profiles, and this is clearly an improvement upon the inviscid theory result.

Figures $4(c, d)$ show profiles of the normal Reynolds stress near a free surface. Apart from the anomalous behaviour of the DNS data between approximately $|z| / L^{*}=0.5$ and $|z| / L^{*}=2$, which again may be attributed to an imperfect ensemble averaging, the theoretical and DNS profiles are in very good agreement. The shape of the viscous theory profile is now even closer to inviscid theory, because the effect of the viscous layer on normal velocity is very weak.

\subsection{Profiles of the turbulence dissipation rate}

Figures 5 and 6 present profiles of the turbulence dissipation rate for a solid wall and for a free surface, respectively. In both figures, the dimensionless time is $U^{*} t / L^{*}=0.1$ and the Reynolds numbers are 54 and 134. The DNS data presented in figure 5 were taken from figures 3.3.9, 3.3.10, 3.3.18 and 3.3.19 of PM93 and those presented in figure 6 were taken from figures 3.2.10, 3.2.11, 3.2 .21 and 3.2 .22 of PM93.

Figures $5(a, b)$ show profiles of the rate of dissipation of tangential Reynolds stress, normalised by their value far from the boundary. The agreement of the viscous theory profiles with the DNS 
data is very good. Even in the viscous layer adjacent to the boundary, where the profiles display large variations because the velocity gradients are large there, the theoretical values show good agreement with the DNS. The inviscid theory, of course, completely fails to model dissipation correctly in this region, giving values which are much lower than observed.

Figures $5(c, d)$ present profiles of dissipation of normal Reynolds stress near a solid wall. The agreement of the viscous theory with the DNS data is good, although dissipation given by the model is slightly smaller in an intermediate region between the boundary and the far-field. Curiously, in this region, the inviscid theory gives a better approximation to the data, particularly in figure $5(c)$ but, as would be expected, the inviscid theory behaves much worse than the viscous theory inside the viscous layer, where the dissipation decreases to zero towards the boundary.

Figures $6(a, b)$ show profiles of the tangential dissipation rate near a free surface. The agreement of the viscous theory and DNS data is good, although there is an overestimation of the maximum in dissipation for $R e^{*}=54$ and a slight overestimation of the viscous layer thickness for $R e^{*}=134$. Nevertheless, it is clear that the profiles of the viscous theory are much better than those of the inviscid theory and there are also signs that the ensemble averaging of the DNS data may not have been perfectly stable. The important finding here, which has implications for the evolution of the tangential Reynolds stress, is that dissipation attains an absolute minimum exactly at the boundary. This contributes towards an intensification of the turbulence at the free surface, a phenomenon noted by Hunt (1984a), PM95a and Walker et al. (1996). Using an inviscid model, Hunt (1984a) attributed this phenomenon to the distortion of vorticity by the upwelling zones that exist near the boundary. PM95a subsequently suggested that this effect is linked with the pressure-strain terms in the turbulent kinetic energy budget near the boundary. While this physical process surely exists, our model supports the idea (put forward by PM95a) that the main factor enhancing the tangential Reynolds stresses at short times is the minimum in tangential dissipation at the boundary: the pressure is intrinsically nonlinear and so scaling arguments of $\S 2$ show that it is negligible at short times. This minimum in dissipation, conjugated with the no-stress boundary condition, also explains the persistence of the 'attached' vortices observed in the experiments of Pan \& Banerjee (1995). The reason why these vortices are two-dimensional, with a vertical axis of rotation, is because the no-stress boundary condition imposes zero horizontal vorticity at the free surface. And they persist for a long time because the dissipation is reduced near the boundary.

Figures $6(c, d)$ present profiles of the normal dissipation rate near a free surface. The agreement of viscous theory with DNS data is very good, particularly for $R e^{*}=134$. Again, a very substantial improvement in the results is achieved inside the viscous layer by the viscous theory, as compared with inviscid theory. In this layer, dissipation is considerably reduced, but not as much as near a solid wall, and attains a finite value at the free surface.

In all the cases analysed above, the behaviour of the turbulence dissipation profiles can be related to the behaviour of the corresponding Reynolds stresses. Basically, dissipation is high in the regions where the velocity gradients are also high. For example, in the viscous layer of a solid wall, the high tangential dissipation results from the steep gradients in tangential Reynolds stress which are required to satisfy the no-slip boundary condition. The fact that normal dissipation is zero at the boundary can be explained using mass conservation and the no-slip boundary condition: since the tangential velocity $u_{\alpha}$ is zero at the boundary, its tangential derivatives are zero, hence by continuity, the normal gradient of the normal velocity is zero. Since the tangential gradients of the normal velocity are also zero, because the boundary is flat, the normal dissipation has to be zero according to (4.5) with $i=3$.

In the viscous layer of a free surface, the reduced tangential dissipation is attributed to the nostress boundary condition, which imposes a zero normal gradient of the tangential Reynolds stress at the boundary. The dissipation is not zero at the boundary because there are still tangential gradients of the tangential velocity. The normal component of dissipation, on the other hand is finite and non-zero at the boundary because the no-stress boundary condition permits the existence of normal gradients of the normal velocity component.

A striking aspect of these comparisons is the success of the RDT model in predicting turbulence dissipation - a process usually associated with the smallest scales of the turbulence. RDT is justified by a time-scale analysis based on the energy-containing eddies, and so is expected to fail for small-scale eddies which evolve on a shorter time scale (Batchelor \& Proudman, 1954). However, in the bounded flows studied here, dissipation near the boundary is dominated by the 
large gradients of the velocities associated with the energy-containing eddies in the viscous layer. The small-scale eddies are of secondary importance in determining the dissipation rate inside the viscous layer. This may explain in part why the normal dissipation rate profiles in figures $5(c$, d) agree less well with the DNS data in an intermediate region between the viscous layer and the far-field. In that region, dissipation has not yet reached its far-field value, but the large-scale velocity has already much weaker gradients, so the velocity gradients of the smaller scales have an increased importance and, as a consequence, RDT is less accurate. In the viscous layer, the role of the smallest scales in the turbulence is primarily that of limiting the magnitude of dissipation through the viscous cutoff wavenumber $k_{\max }$, but these small scales are relatively unimportant for determining the actual shape of the dissipation profiles.

\subsection{Time evolution of dissipation}

Figure 7 shows profiles of the tangential and normal dissipation rates near a solid wall and a free surface, for a Reynolds number of 134, at the times $U^{*} t / L^{*}=0,0.01,0.05,0.1$ and 0.15 , as calculated from viscous theory. The DNS data presented in the same figure were taken from figures 2 and 3 of PM95b, and refer to the much later time $U^{*} t / L^{*}=2$.

The evolution of the theoretical solutions with time can be seen. At $t=0$, the dissipation rates begin by being equal to the dissipation rates calculated from inviscid theory. At the boundary, the tangential dissipation component is twice its value in the far-field, and the dissipation smoothly decays to its far-field value as one moves away from the boundary. The normal dissipation has the same value at the boundary and in the far-field, with a minimum in between located at about $z / L^{*}=0.05$.

As time progresses, the influence of the viscous layer spreads over increasingly larger distances, and the theoretical profiles come to resemble the DNS profiles much closer, in particular regarding the location of maxima and minima. The match is not perfect, however, because while the model gives a reasonable prediction of the thickness of the viscous layer at a model time of $U^{*} t / L^{*}=0.1$ or $U^{*} t / L^{*}=0.15$ (in particular in figures $7(b-d)$ ), the DNS curves tend to decay much slower towards their asymptotic value far from the boundary than the theoretical curves. We attribute this behaviour to the additional diffusive effect of the turbulent transport (neglected in the model), which can be felt far outside the viscous layer.

Near a solid wall, tangential dissipation (figure $7(a)$ ) is enhanced inside the viscous layer. As the viscous layer thickens, the maximum in the dissipation, which occurs at the boundary, is reduced as the velocity gradients in the viscous layer reduce. A region where dissipation is lower than in the inviscid profile exists at the edge of the viscous layer. Qualitatively, the DNS profile at the late time, $U^{*} t / L^{*}=2$, displays similar features, as follows. The maximum at the boundary is higher than would be expected from inviscid theory, consistently with the existence of a viscous boundary layer. However, the value at the maximum is considerably lower than at early times, and the region of lowered dissipation noted in the theoretical profiles is more pronounced and spread over a much wider distance.

The time evolution of the normal dissipation shown in figure $7(b)$ begins with a local maximum very near the boundary. This maximum is progressively eroded because of the growth of the viscous layer and the constraint that normal dissipation is zero exactly at the boundary. Nevertheless, a residual maximum in curvature persists in the theoretical profiles up to the latest time considered, and this maximum may also be observed in the DNS data. At $U^{*} t / L^{*}=0.15$, the theoretical profile departs slightly from its correct value far from the boundary, because the truncation error has become too large due to the power series of the solution becoming nearly non-asymptotic (see $\S 4.2)$.

For a free surface, the tangential dissipation (figure $7(c)$ ) begins by having a maximum near the boundary, and a sharp reduction exactly at the boundary, associated with the no-stress condition. As time progresses, these features become smoothed. The maximum decreases and moves away from the boundary and the minimum also decreases slightly, becoming distinctly lower than the dissipation value far from the boundary. The thickness of the viscous layer, as identified by the dissipation maximum, is in good agreement with DNS data for a model time of $U^{*} t / L^{*}=0.15$.

The normal dissipation (figure $7(d)$ ) initially has a local maximum at the boundary, followed by a minimum before tending to the far-field value. The maximum is soon eroded as the viscous layer grows, so that at the latest time considered in the model, dissipation has become approxi- 
mately constant in the viscous layer. Again, there is a certain deal of qualitative agreement of the theoretical profiles at $U^{*} t / L^{*}=0.1$ or 0.15 with the DNS profile.

Except for a considerably higher maximum in tangential dissipation at the boundary in the case of a solid wall, and a generally faster decay of the curves towards their far-field values as one moves away from the boundary, which we argue is due to the neglect of turbulent transport, the dissipation profiles presented in figure 7 for the viscous theory at $U^{*} t / L^{*}=0.1$ or $U^{*} t / L^{*}=0.15$ resemble the profiles obtained from the DNS at the late time $U^{*} t / L^{*}=2$. This happens partly because the boundary conditions at $z=0$ are independent of time and also because the viscous layer does not grow indefinitely, but rather its growth is halted by nonlinear effects when $\delta / L \approx R e^{-1 / 2}$ (Hunt \& Graham, 1978). The same nonlinear effects are also responsible for the main differences between the theoretical and DNS profiles, namely the diffusion of relatively sharp features like the minimum in tangential dissipation in figure $7(a)$.

Nevertheless, the similarities between the RDT and the DNS at late times remain striking, which suggests the concept of a total diffusion of the viscous boundary layer, akin to the total shear introduced by Townsend (1976), and used more recently by Mann (1994), for matching the results of RDT and measurements of equilibrium shear-flow turbulence. Qualitatively, equilibrium shear-flow turbulence and initially isotropic turbulence suddenly distorted by a constant shear are quite different. In equilibrium turbulence, vorticity tilting and stretching by shear is balanced by turbulent transport and viscous diffusion. In rapid distortion by shear, the turbulence is nonstationary, and dominated by vorticity tilting and stretching. Townsend noted that the first kind of turbulence resembled the second if, in his rapid-distortion model, the total shear since the beginning of the distortion was chosen appropriately.

The current model shows that homogeneous decaying turbulence near a solid or free boundary at relatively long times qualitatively resembles turbulence distorted by the same boundary at a given total diffusion of the viscous boundary layer. This total diffusion corresponds to a time $T_{\nu}$ when the model breaks down due to the growing relevance of neglected physical processes, such as turbulent transport. The main effect of these processes seems to be to arrest the fast evolution of the turbulence at an early distortion stage, changing it only slowly at subsequent times.

\section{Conclusions}

The rapid-distortion model of Hunt \& Graham (1978) has been extended to treat the early development of shear-free turbulence near a suddenly introduced solid wall or free surface, taking full account of viscous processes, including the dynamics of the viscous boundary layer. The model is linear and assumes the turbulence to be initially homogeneous and isotropic everywhere, and to remain so far from the boundary. The turbulent velocity field is expressed as a superposition of Fourier modes and then statistics of the velocity field are obtained, given the energy spectrum of the turbulence far from the boundary. The velocity components induced by the boundary are expanded in power series of a small parameter, and terms in the series are considered up to the point where they become comparable with the neglected nonlinear effects. The model is formally valid at short times when (i) the nonlinear terms in the equations of motion are negligible and (ii) the power series of the solution for the turbulent velocity are asymptotic. Condition (i), which is well known from other rapid distortion studies, ensures in this particular case that viscous diffusion dominates over turbulent transport in the dynamics of the viscous layer. Condition (ii), on the other hand, ensures that the viscous layer can be treated as a thin boundary layer, and requires it to be much thinner than the smallest scales in the turbulence. The model makes use of a turbulence

spectrum with a viscous cutoff in order to be dynamically consistent. This spectrum was found to lead to a correct dependence of the dimensionless dissipation on the Reynolds number, as compared with recent DNS data.

Reynolds stress and turbulence dissipation profiles were then calculated. The model was able to reproduce the essential observed differences between a solid wall and a free surface. Regarding dissipation, these differences are: dissipation of the tangential Reynolds stress is enhanced near a solid wall, while it is reduced near a free surface; dissipation of the normal Reynolds stress tends to zero near a solid wall, while it is slightly reduced to a non-zero value near a free surface. Results at short times were seen to be in good quantitative agreement with available DNS data, and also to reproduce the qualitative features of DNS data (namely the location of maxima and minima in the profiles) at later times, provided that the time used in the model was appropriately chosen. 
This suggests that the structure of the turbulence statistics and their differences for each type of boundary are essentially determined by the linear dynamics of the viscous boundary layer.

This study clarifies why the inviscid Hunt \& Graham theory can be applied, with relative success, to the prediction of the Reynolds stress profiles near free surfaces at relatively long times (cf. Brumley and Jirka, 1987; Perot \& Moin, 1995a; Walker et al., 1996). On the one hand, a free surface introduces a relatively weak viscous correction to the essentially inviscid flow associated with the blocking effect of the boundary. That correction reduces only slightly the tangential Reynolds stresses at the boundary so that the boundary condition can be enforced. On the other hand, the tangential dissipation at the free surface is lower than in the bulk of the flow, as referred above, and this leads to a slow enhancement over time of the tangential Reynolds stresses which counteracts the reduction associated with the boundary condition. Because of these two opposing effects, the inviscid solution remains valid for times longer than expected.

Ultimately, the viscous layer stops growing, and the effect of dissipation supersedes that of the boundary condition, leading to the tangential Reynolds stresses at the free surface becoming greater than those predicted from inviscid theory. Alternative explanations for this phenomenon have been suggested, involving energy transfer from the normal to the tangential velocity components by the pressure-strain terms in the turbulent kinetic energy equation. However, our model supports the idea, put forward by PM95a, that the minimum in the dissipation profile at the surface plays a crucial role in this phenomenon, at least at the initial stages, because pressure is an intrinsically nonlinear quantity which was seen to be negligible at short times.

Turbulence dissipation also has implications for air-water gas transfer. The speed at which gases are transported across an air-water interface where the water is in turbulent motion is determined by: how thin the viscous boundary layer is forced to remain by the turbulence and how fast the turbulent motions replace old fluid near the surface by new fluid (Theofanous, 1984). This surface renewal is determined by the velocity and length scales of the turbulence, and also by the divergence of the flow at the surface, particularly at high Reynolds numbers (Hunt, 1984b). Indirectly, the dissipation profile near a free surface promotes surface renewal by increasing the value of the turbulence velocity scale there. At the same time, the dissipation rate of the normal Reynolds stress, $\varepsilon_{33}$, is linked with the surface divergence, through definition (4.5) with $i=3$, because at $z=0$ the tangential derivatives of the normal velocity are zero and the normal derivative of the vertical velocity can be related, using continuity, to the horizontal divergence. The normal dissipation rate is thus proportional to the mean square surface divergence, and so promotes surface renewal.

We now present some more general conclusions. One of the main findings of this study is that dissipation near a shear-free boundary is primarily determined by the velocity gradients corresponding to the rapid variation across the viscous boundary layer of the energy containing eddies. The small scales in the turbulence were seen to be important mainly for limiting the magnitude of dissipation, but not for determining the shape of the dissipation profiles. Therefore, the reason why rapid-distortion theory is in this case appropriate for calculating dissipation is because the boundary introduces a strong inhomogeneity in the flow.

Another key conclusion is that the shape of the dissipation profiles in turbulence near a solid wall or a free surface is essentially determined by the linear dynamics of the viscous boundary layer, even at late times. The effect of nonlinear processes seems to be primarily to arrest the growth of the viscous boundary layer on the one hand and to slightly diffuse the features in the profiles on the other.

We are grateful to Paviz Moin and Blair Perot for providing a copy of the technical report TF-60 of the Thermosciences Division, Department of Mechanical Engineering, Stanford University. M.A.C.T. acknowledges the financial support of the Portuguese Fundação para a Ciência e Tecnologia (FCT), which funded this work under grant PraxisXXI/BD/9401/96.

\section{Appendix. Expressions of the functions $M_{i k}$}

\section{(a) Solid wall}

For a solid wall, the functions $M_{i k}(\boldsymbol{k}, z, t)$ are defined as follows:

$$
M_{\alpha \alpha}=\mathrm{e}^{\mathrm{i} k_{3} z}-\left[\operatorname{erf}\left(\frac{z}{\delta}\right)+1\right]
$$




$$
\begin{aligned}
& M_{\alpha 3}=-\frac{\mathrm{i} k_{\alpha}}{k_{12}}\left[1-\frac{\delta}{\pi^{1 / 2}}\left(1+\frac{\delta k_{12}}{\pi^{1 / 2}}\right)\left(\mathrm{i} k_{3}-k_{12}\right)\right]\left[\mathrm{e}^{k_{12} z}-\left(\operatorname{erf}\left(\frac{z}{\delta}\right)+1\right)\right], \quad \alpha=1,2 \\
& M_{33}=\mathrm{e}^{\mathrm{i} k_{3} z}-\mathrm{e}^{k_{12} z}+\frac{\delta}{\pi^{1 / 2}}\left(1+\frac{\delta k_{12}}{\pi^{1 / 2}}\right)\left(\mathrm{i} k_{3}-k_{12}\right)\left(\mathrm{e}^{k_{12} z}-1\right)-\left(\mathrm{i} k_{3}-k_{12}\right)\left(1+\frac{\delta k_{12}}{\pi^{1 / 2}}+\frac{\delta^{2} k_{12}^{2}}{\pi}\right) \\
& \times \delta\left[\frac{z}{\delta}\left(\operatorname{erf}\left(\frac{z}{\delta}\right)+1\right)+\frac{1}{\pi^{1 / 2}}\left(\mathrm{e}^{-z^{2} / \delta^{2}}-1\right)\right] .
\end{aligned}
$$

The remaining elements of the $M_{i k}$ matrix are zero.

Using (4.4), the Reynolds stresses may be expressed in terms of the undistorted turbulence spectrum $\Phi_{i j}^{(H)}$ and using (4.5), (4.6) and (4.7), the dissipation rates may be expressed in terms of the undistorted turbulence spectrum.

The resulting expressions may be further simplified by expressing the integrals involved in spherical polar coordinates and using (4.8). This enables analytical integration in one of the axial variables and the triple integrals become double integrals. That is the simplest form they can take before numerical evaluation, which is carried out using the Mathematica numerical package.

\section{(b) Free surface}

For a free surface, the non-zero components of the $M_{i k}(\boldsymbol{k}, z, t)$ tensor can be written as follows:

$$
\begin{aligned}
& M_{\alpha \alpha}=\mathrm{e}^{\mathrm{i} k_{3} z}-\mathrm{i} k_{3} \delta\left[\frac{z}{\delta}\left(\operatorname{erf}\left(\frac{z}{\delta}\right)+1\right)+\frac{1}{\pi^{1 / 2}} \mathrm{e}^{-z^{2} / \delta^{2}}\right] \\
& M_{\alpha 3}=-\frac{\mathrm{i} k_{\alpha}}{k_{12}}\left(1+\frac{\delta^{2} k^{2}}{4}\right)\left\{\mathrm{e}^{k_{12} z}-k_{12} \delta\left[\frac{z}{\delta}\left(\operatorname{erf}\left(\frac{z}{\delta}\right)+1\right)+\frac{1}{\pi^{1 / 2}} \mathrm{e}^{-z^{2} / \delta^{2}}\right]\right\}, \quad \alpha=1,2 \\
& M_{33}=\mathrm{e}^{\mathrm{i} k_{3} z}-\mathrm{e}^{k_{12} z}+\frac{\delta^{2} k^{2}}{4}\left(1-\mathrm{e}^{k_{12} z}\right)+\left(1+\frac{\delta^{2} k_{12}^{2}}{4}\right) \delta^{2} k^{2} \\
& \times\left[\frac{z^{2}}{2 \delta^{2}}\left(\operatorname{erf}\left(\frac{z}{\delta}\right)+1\right)+\frac{z}{2 \pi^{1 / 2} \delta} \mathrm{e}^{-z^{2} / \delta^{2}}+\frac{1}{4} \operatorname{erf}\left(\frac{z}{\delta}\right)\right] .
\end{aligned}
$$

Again, using (4.4), (4.5), (4.6) and (4.7), the Reynolds stresses and the dissipation rates may be expressed in terms of the undistorted turbulence spectrum $\Phi_{i j}^{(H)}$.

The integrals contained in those expressions may then be expressed in spherical polar coordinates and integrated analytically over one of the axial variables, becoming double integrals, and then evaluated numerically using Mathematica.

\section{REFERENCES}

Batchelor, G. K. 1953 The Theory of Homogeneous Turbulence. Cambridge University Press.

Batchelor, G. K. And Proudman, I. 1954 The effect of rapid distortion of a fluid in turbulent motion. Quart. J. Mech. Appl. Math., 7, 83-103.

Brumley, B. H. And JiRkA, G. H. 1987 Near-surface turbulence in a grid-stirred tank. J. Fluid Mech., $183,235-263$.

Hallbäck, M., Groth, J. And Johansson, A. V. 1990 Algebraic model for nonisotropic turbulent dissipation rate in Reynolds stress closures. Phys. Fluids A, 2, 1859-1866.

Hunt, J. C. R. 1973 A theory of flow round two-dimensional bluff bodies. J. Fluid Mech., 61, 625-706.

Hunt, J. C. R. 1984a Turbulence structure in thermal convection and shear-free boundary layers. J. Fluid Mech., 138, 161-184.

Hunt, J. C. R. $1984 b$ Turbulence structure and turbulent diffusion near gas-liquid interfaces. In Gas transfer at water interfaces, edited by W. Brutsaert and G.H. Jirka, D. Reidel Publishing Company.

Hunt, J. C. R. And Graham, J. M. R. 1978 Free stream turbulence near plane boundaries. J. Fluid Mech., 84, 209-235.

Jiménez, J., Wray, A. A., Saffman, P. G. And Rogallo, R. S. 1993 The structure of intense vorticity in isotropic turbulence. J. Fluid Mech., 255, 65-90.

ManN, J. 1994 The spatial structure of neutral atmospheric surface-layer turbulence. J. Fluid Mech., 273, $141-168$.

Pan, Y. And BanerJee, S. 1995 A numerical study of free-surface turbulence in channel flow. Phys. Fluids, 7, 1649-1664.

Perot, B. AND MoIn, P. 1993 Shear-free turbulent boundary layers: physics and modeling. Report No. TF-60, Thermosciences Division, Department of Mechanical Engineering, Stanford University.

Perot, B. And Moin, P. 1995 a Shear-free turbulent boundary layers. Part 1. Physical insights into near-wall turbulence. J. Fluid Mech., 295, 199-227. 
Perot, B. And Moin, P. $1995 b$ Shear-free turbulent boundary layers. Part 2. New concepts for Reynolds stress transport equation modelling of inhomogeneous flows. J. Fluid Mech., 295, 229-245.

Tennekes, H. And Lumley, J. L. 1972 A first course in turbulence. The MIT Press.

Theofanous, T. G. 1984 Conceptual models of gas exchange. In Gas transfer at water surfaces, edited by W. Brutsaert and G.H. Jirka, D. Reidel Publishing Company.

Thomas, N. H. And Hancock, P. E. 1977 Grid turbulence near a moving wall. J. Fluid Mech., 82, 481-496.

Townsend, A. A. 1976 The structure of turbulent shear flow, 2nd edition. Cambridge University Press.

Uzkan, T. And Reynolds, W. C. 1967 A shear-free turbulent boundary layer. J. Fluid Mech., 28, $803-821$.

Walker, D. T., Leighton, R. I. And Garza-Rios, L. O. 1996 Shear-free turbulence near a flat free surface. J. Fluid Mech., 320, 19-51.

Wang, L., Chen, S., Brasseur, J. G. and Wyngaard, J. C. 1996 Examination of hypotheses in the Kolmogorov refined turbulence theory through high-resolution simulations. Part 1 . Velocity field. $J$. Fluid Mech., 309, 113-156. 


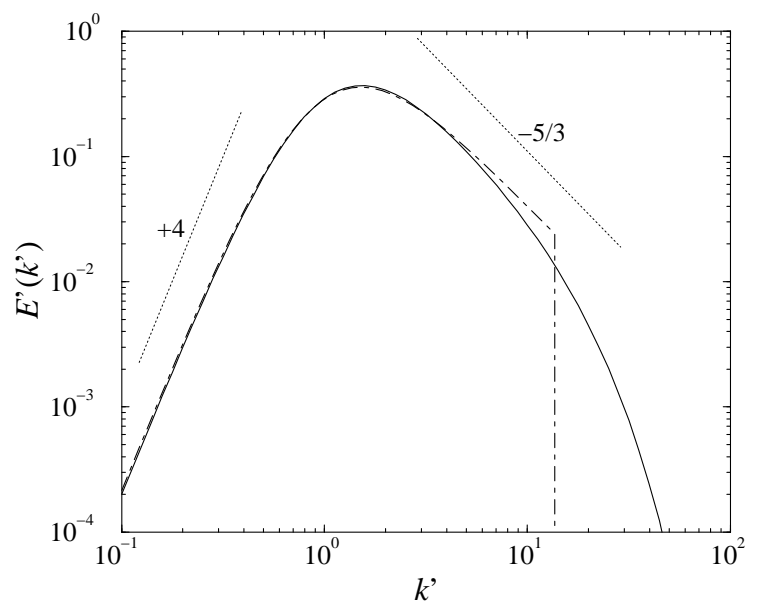

FiguRE 1. Dimensionless energy spectrum as a function of the dimensionless wavenumber, at $R e=70$. Solid line, complete spectrum (4.18); Dash-dotted line, truncated Von Kármán spectrum (4.20). 


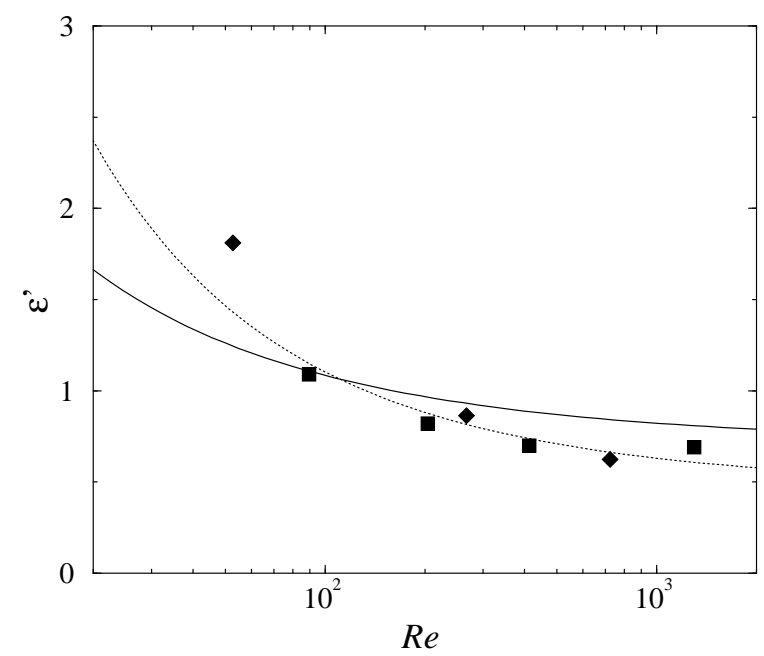

FIGURE 2. Variation of the dimensionless dissipation with the Reynolds number. Solid line, theory using the truncated spectrum (4.20) and $\alpha=1.5$; dotted line, theory using the complete spectrum (4.18) and $\alpha=2$; squares, data of Jiménez et al. (1993); diamonds, data of Wang et al. (1996). 

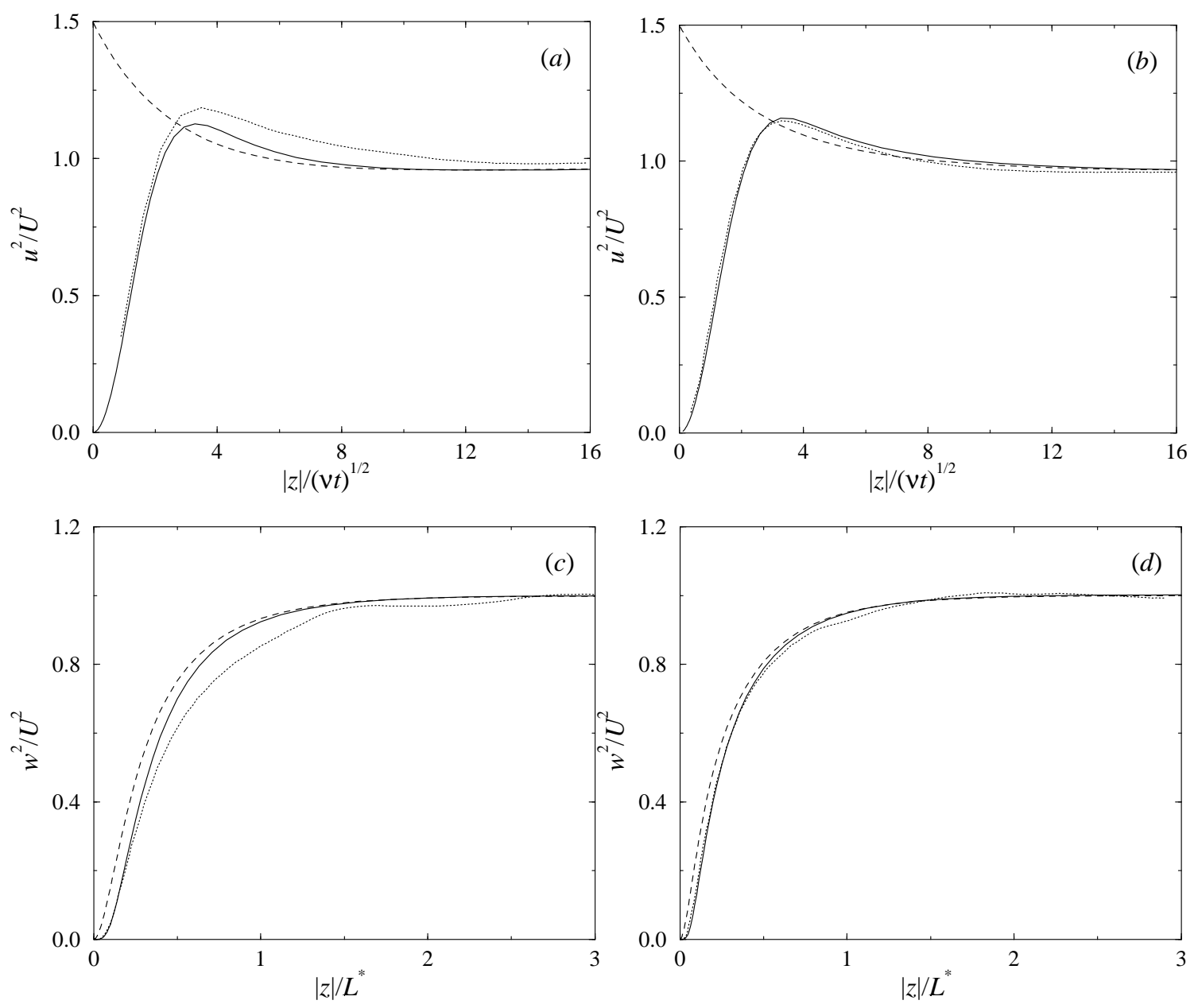

Figure 3. Reynolds stress profiles near a solid wall at $U^{*} t / L^{*}=0.1$. Solid line, viscous theory; dashed line, inviscid theory; dotted line, DNS data. (a) tangential component, $R e^{*}=54(b)$ tangential component, $R e^{*}=134(c)$ normal component, $R e^{*}=54(d)$ normal component, $R e^{*}=134$. 

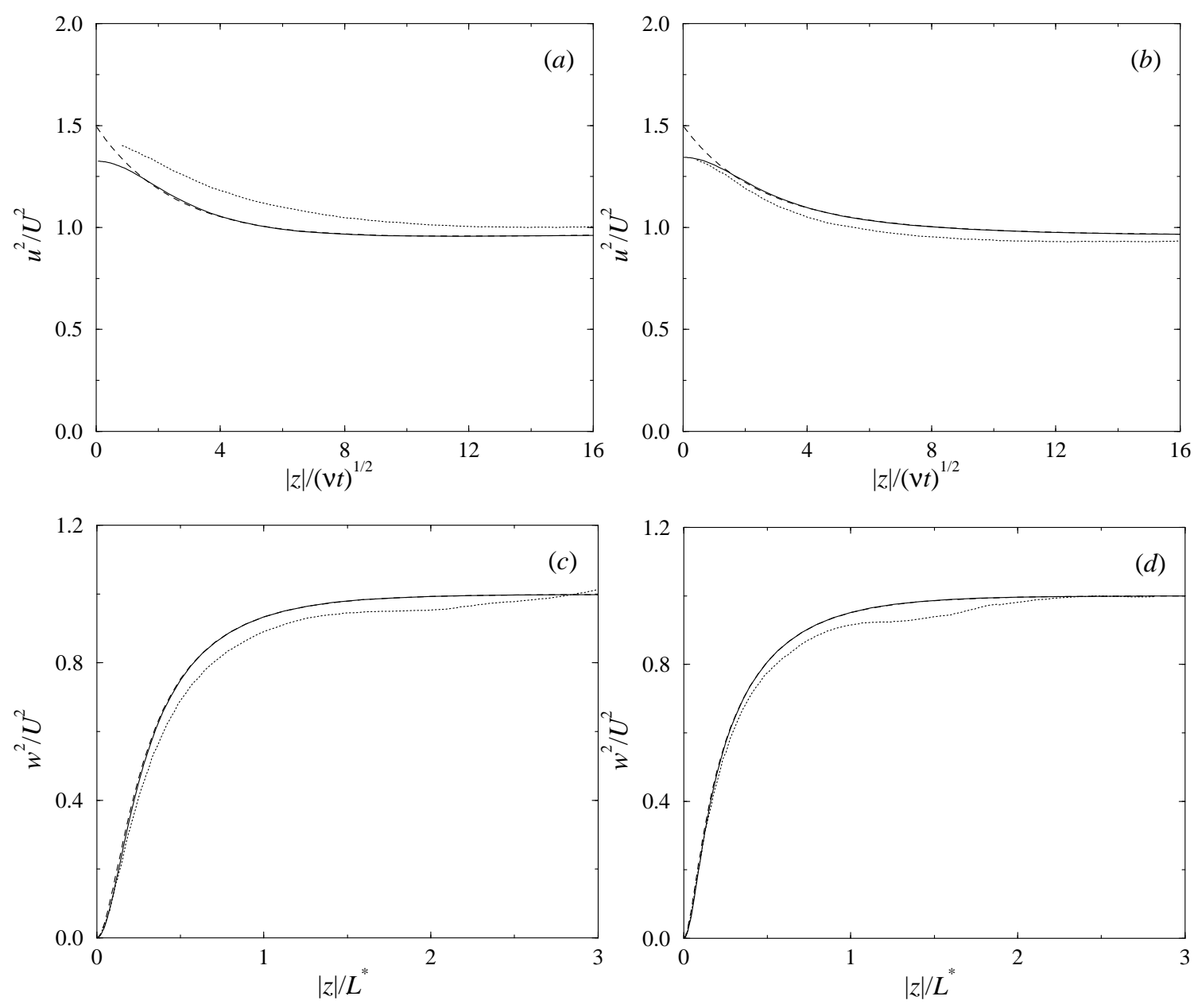

FIgURE 4. Reynolds stress profiles near a free surface at $U^{*} t / L^{*}=0.1$. Solid line, viscous theory; dashed line, inviscid theory; dotted line, DNS data. (a) tangential component, $R e^{*}=54(b)$ tangential component, $R e^{*}=134(c)$ normal component, $R e^{*}=54(d)$ normal component, $R e^{*}=134$. 

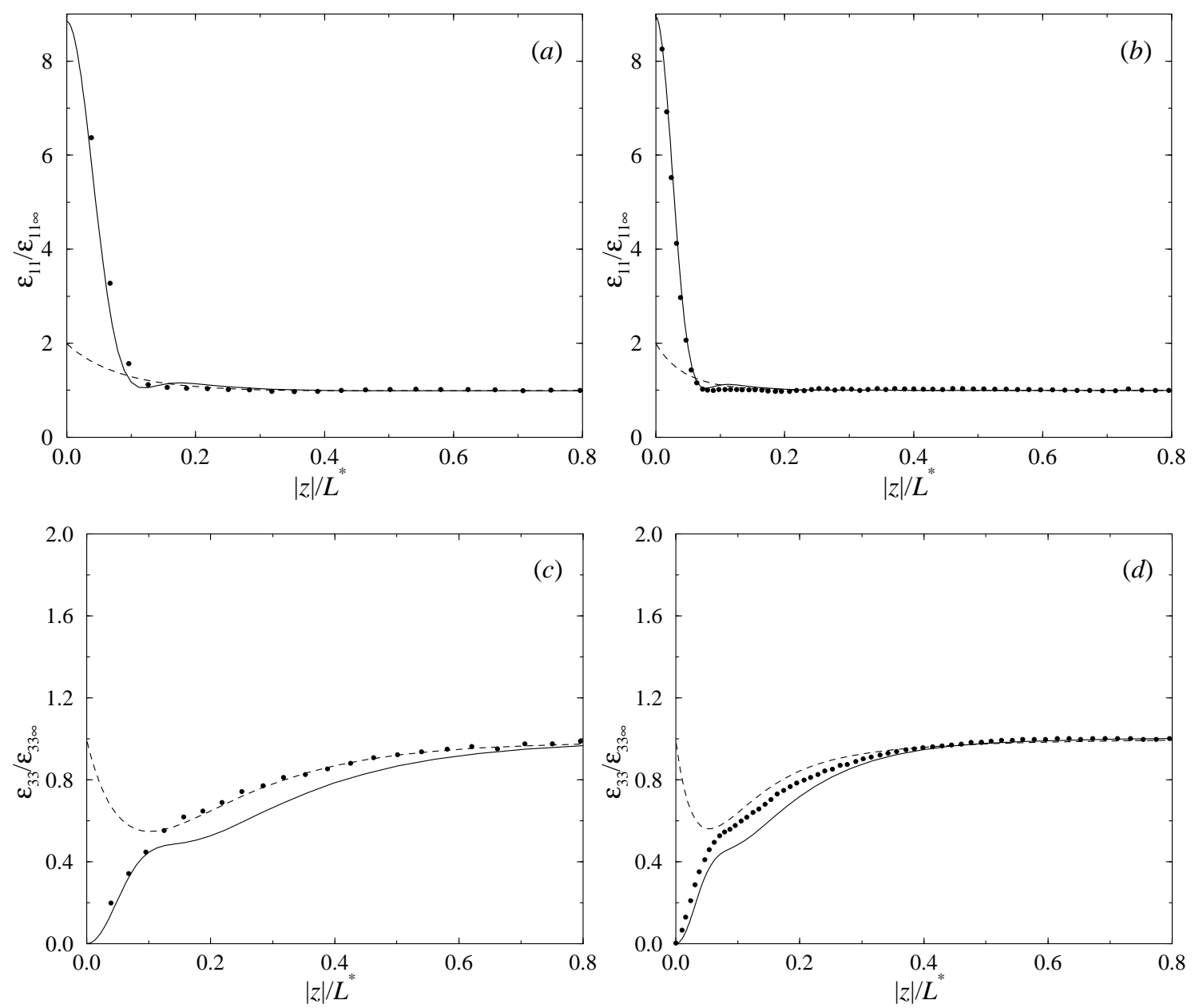

Figure 5. Dissipation rate profiles near a solid wall at $U^{*} t / L^{*}=0.1$. Solid line, viscous theory; dashed line, inviscid theory; circles, DNS data. (a) tangential component, $R e^{*}=54(b)$ tangential component, $R e^{*}=134(c)$ normal component, $R e^{*}=54(d)$ normal component, $R e^{*}=134$. 

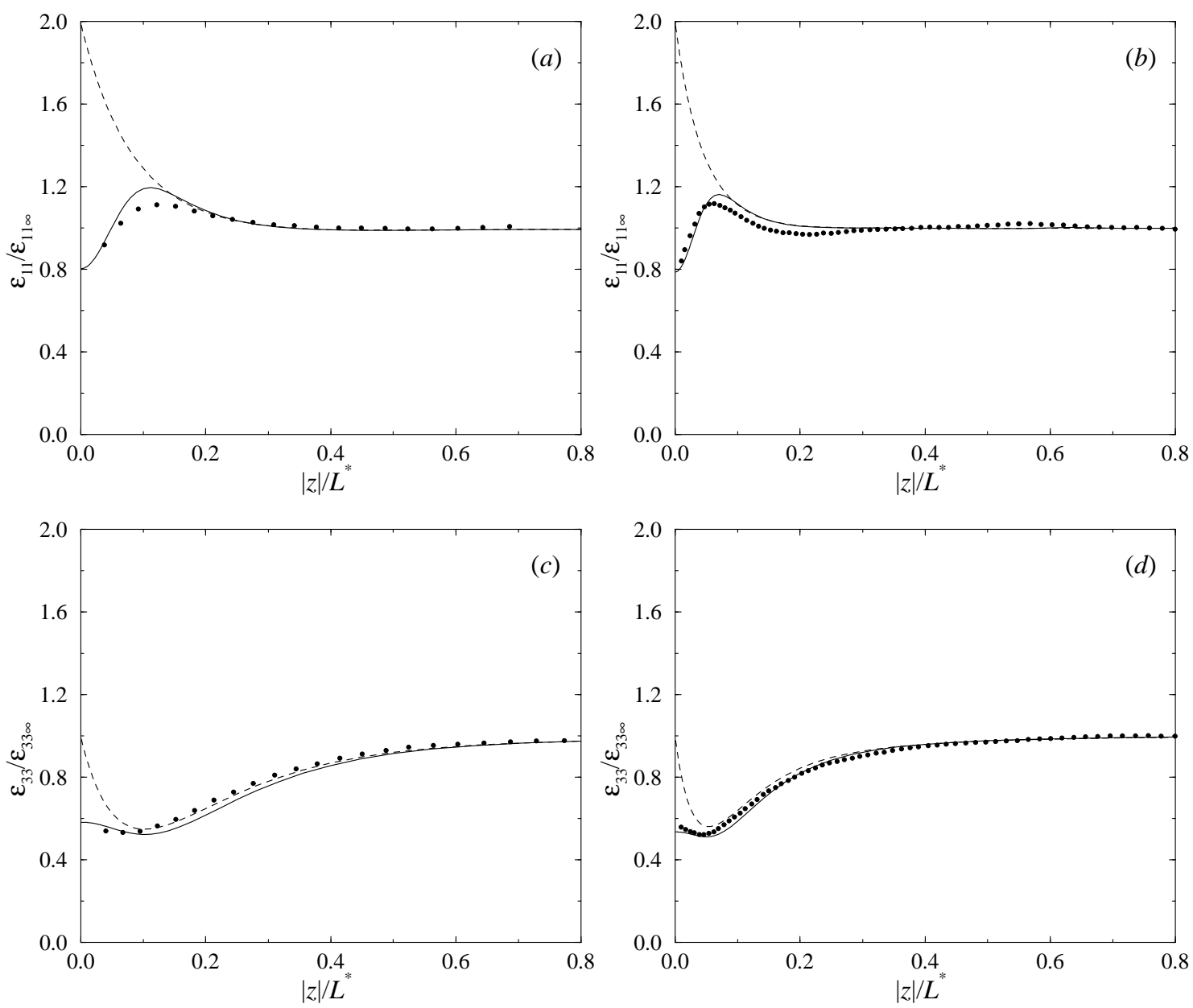

Figure 6. Dissipation rate profiles near a free surface at $U^{*} t / L^{*}=0.1$. Solid line, viscous theory; dashed line, inviscid theory; circles, DNS data. (a) tangential component, $R e^{*}=54(b)$ tangential component, $R e^{*}=134(c)$ normal component, $R e^{*}=54(d)$ normal component, $R e^{*}=134$. 

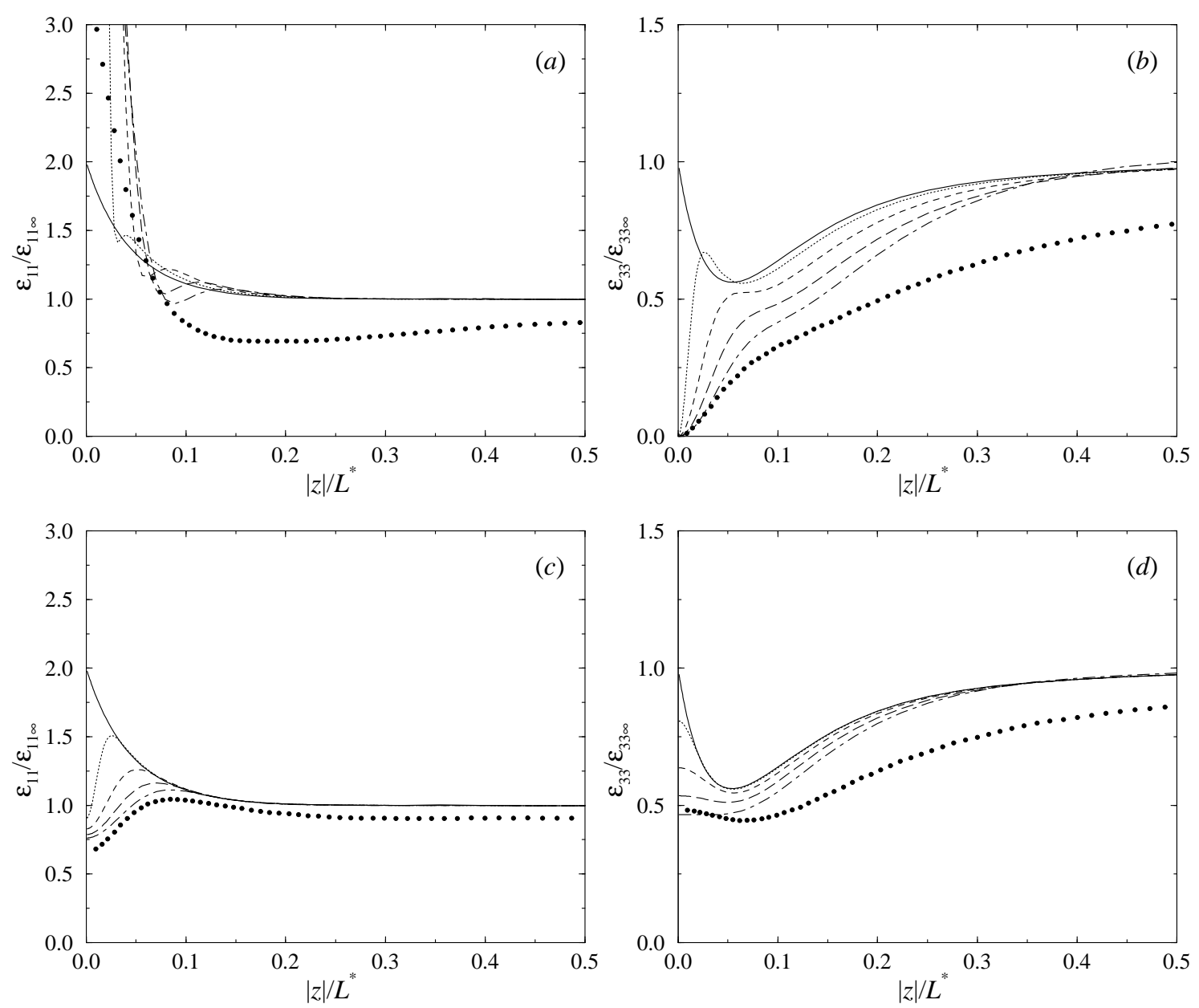

FIgURE 7. Dissipation rate profiles for $R e^{*}=134$ at various times. Viscous theory at: solid line, $U^{*} t / L^{*}=0$; dotted line, $U^{*} t / L^{*}=0.01$; dashed line, $U^{*} t / L^{*}=0.05$; long-dashed line, $U^{*} t / L^{*}=0.1$; dash-dotted line, $U^{*} t / L^{*}=0.15$; circles, DNS data at $U^{*} t / L^{*}=2$. (a) solid wall, tangential component $(b)$ solid wall, normal component $(c)$ free surface, tangential component $(d)$ free surface, normal component. 